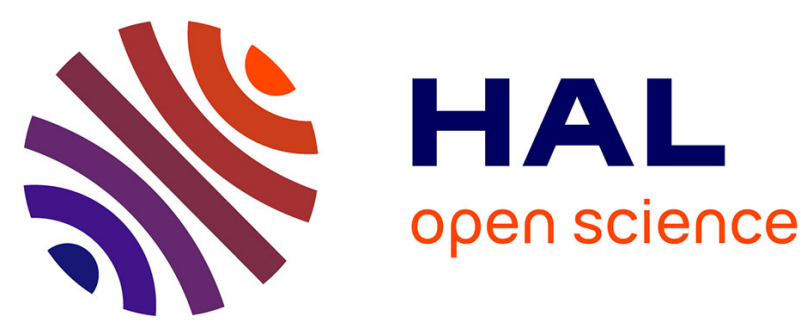

\title{
Thickness and angular dependence of the magnetocurrent of hot electrons in a magnetic tunnel transistor with crossed anisotropies
}

\author{
Michel Hehn, C. Vautrin, D. Lacour, G. Sala, Y. Lu, F. Montaigne
}

\section{To cite this version:}

Michel Hehn, C. Vautrin, D. Lacour, G. Sala, Y. Lu, et al.. Thickness and angular dependence of the magnetocurrent of hot electrons in a magnetic tunnel transistor with crossed anisotropies. Physical Review B: Condensed Matter and Materials Physics (1998-2015), 2017, 96 (17), pp.174426. 10.1103/PhysRevB.96.174426 . hal-01686930

\section{HAL Id: hal-01686930 \\ https://hal.univ-lorraine.fr/hal-01686930}

Submitted on 17 Jan 2018

HAL is a multi-disciplinary open access archive for the deposit and dissemination of scientific research documents, whether they are published or not. The documents may come from teaching and research institutions in France or abroad, or from public or private research centers.
L'archive ouverte pluridisciplinaire HAL, est destinée au dépôt et à la diffusion de documents scientifiques de niveau recherche, publiés ou non, émanant des établissements d'enseignement et de recherche français ou étrangers, des laboratoires publics ou privés. 


\title{
Thickness and angular dependence of the magnetocurrent of hot electrons in a magnetic tunnel transistor with crossed anisotropies
}

\author{
C. Vautrin, D. Lacour, G. Sala, Y. Lu, F. Montaigne, and M. Hehn \\ Institut Jean Lamour, CNRS, Nancy-Université, B.P. 70239, F-54506 Vandoeuvre lès Nancy, France
}

(Received 3 September 2017; published 20 November 2017)

\begin{abstract}
We have studied the thickness and angular dependence of the magnetocurrent of hot electrons in a magnetic tunnel transistor (MTT) with crossed magnetic anisotropies. In a first step, we show that the magnetocurrent increases with ferromagnetic layer thickness as for MTTs with collinear magnetic configurations. The maximum magnetocurrent value is obtained to be $85 \%$, which is close to the theoretical maximum value of $100 \%$ for MTTs with crossed magnetic configurations. In a second step, we demonstrate that we are able to reproduce both current vs field direction and current vs field intensity measurements in a framework taking into account a reduced number of magnetic parameters and a simple cosine dependence of the hot-electron current on the angle between magnetizations.
\end{abstract}

DOI: 10.1103/PhysRevB.96.174426

\section{INTRODUCTION}

Combining hot-electron spin-filtering effects in ferromagnetic materials with the energy selectivity in spin-valve transistors led Monsma et al. to demonstrate huge magnetocurrent (MC) contrasts at room temperature [1]. The high $\mathrm{MC}$ value reached through the spin filtering of hot electrons motivated the development of magnetic tunnel transistors (MTTs), in which the injection of hot electrons is done using tunnel barriers [2-5] instead of Schottky barriers [1,6] simplifying the device fabrication. Only collinear magnetization configurations have been studied in the past. Very recently, we reported the study of a MTT with crossed anisotropies by engineering the crystallographic structure and the magnetic properties of the spin-valve ferromagnetic layers $[7,8]$.

In this study, we take advantage of the crossed magnetizations geometry to check the cosine angular dependence of the hot-electron current on the angle between both magnetizations as stated by Applebaum's 2011 review paper [9]. Furthermore, as for MTTs with collinear magnetic configurations, we report the magnetocurrent as a function of the magnetic layer thickness that increases with layer thickness up to $85 \%$, slightly lower than $100 \%$, the maximum theoretical magnetocurrent that can be reached in the crossed magnetizations configuration.

\section{EXPERIMENTAL DETAILS}

The MTT structure used in this study is identical to the one used in Refs. [7,8]. It is based on a multilayer grown by sputtering using an Alliance Concept DP850 UHV machine on a HF-prepared low-resistance $\mathrm{Si}$ with stacking $\mathrm{Si} / \mathrm{Cu}(5) / \mathrm{Ta}(1) / \mathrm{Cu}(5) / \mathrm{Ni}(0.6) /[\mathrm{Co}(0.2) / \mathrm{Ni}(0.6)]_{N} / \mathrm{Cu}(3.5) /$ $\mathrm{Co}(3) / \mathrm{MgO}(2.7) / \mathrm{Cu}(20) / \mathrm{Pt}(5)$ (sketched in Fig. 1, thickness in brackets in nanometers). In order to check the magnetocurrent, the samples are processed using clean-room facilities to make electrical contacts to define the emitter on the top $\mathrm{Cu} / \mathrm{Pt}(5)$, the base on the metallic $\mathrm{Cu} / \mathrm{Pt} /[\mathrm{Co} / \mathrm{Ni}]_{5} /$ $\mathrm{Cu} / \mathrm{Co}$, and the collector on the Si substrate. A schematic of the electrical connections is given in Fig. 1. The electrical transport measurements were done using an ARS cryocooler with a rotating electromagnet that can reach an applied field of $2 \mathrm{~T}$. A current of electrons, denoted $I_{\mathrm{e}}$, is injected from the $\mathrm{Cu} / \mathrm{MgO} / \mathrm{Co}$ magnetic tunnel junction into the $\mathrm{Co} / \mathrm{Cu} /[\mathrm{Co} / \mathrm{Ni}]$ spin valve. The hot electrons that conserve their energy above the $\mathrm{Cu} / \mathrm{Si}$ Schottky barrier height, typically around $0.7 \mathrm{eV}$, are collected into the Si substrate. This current of hot electrons is denoted $I_{\mathrm{c}}$.

Since our crossed magnetizations geometry cannot provide the antiparallel configuration, we define the magnetocurrent contrast between the crossed and the parallel magnetic configurations as

$$
\mathrm{MC}^{\perp}=100\left(\frac{I_{C}^{P}-I_{C}^{\perp}}{I_{C}^{\perp}}\right),
$$

with $I_{C}^{P}$ and $I_{C}^{\perp}$ being, respectively, the collected currents in the parallel and in the crossed magnetic configurations. The parallel configuration is obtained for a magnetic field equal to $20 \mathrm{kOe}$ applied in the sample plane [see Fig. 2(a)] while the crossed configuration is obtained by decreasing the field to zero.

\section{RESULTS}

The collected current in the semiconductor has been measured as a function of the applied voltage for the spin valve in the parallel magnetic configuration or in a crossed magnetic configuration as shown in Fig 2(b). As expected, the current is higher in the case of the parallel magnetic configuration. Indeed, a hot electron has a larger mean-free path if its spin is parallel to the magnetization of the ferromagnetic layer. An electron having a spin of opposite direction suffers inelastic scattering events and loses a part of its energy. As a consequence, if the spin valve is in the parallel configuration, the electrons with the spin oriented parallel to the direction of both magnetizations can cross the spin valve with only a small energy loss. On the contrary, an electron with a spin in the opposite direction will lose too much energy to overcome the Schottky barrier. Thus, a 100\% spin-polarized current can be collected. If the spin valve is in a crossed configuration, an electron will lose a part of its energy regardless of the 


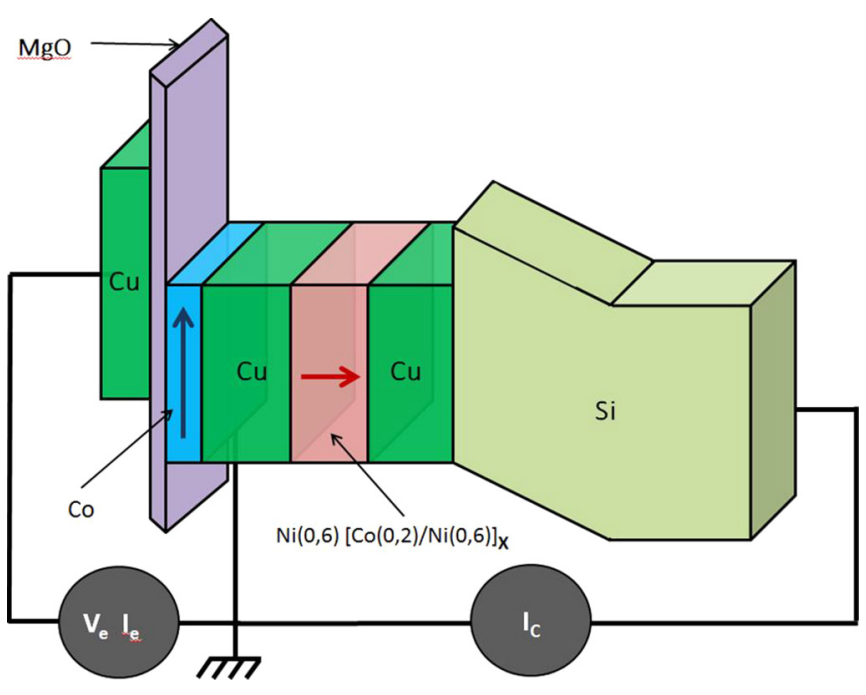

FIG. 1. Schematic band diagram of MTT on N-Si for hot-electron injection. Voltage across the $\mathrm{MgO}$ tunnel barrier controls the injection energy of the hot electrons.

spin direction: The current collected is between the collected current in the parallel and antiparallel configurations.

Then, the $\mathrm{MC}^{\perp}$ versus applied voltage is obtained by the normalized difference of both curves using Eq. (1). As reported in Fig. 2(c), $\mathrm{MC}^{\perp}$ decreases slowly from $87 \%$ to $75 \%$ when the applied voltage decreases from -0.69 to $-1 \mathrm{~V}$. This trend is the one expected for hot electrons since the contrast between the up and the down spin channels decreases when the electron energy increases well above the Schottky barrier height. However, and surprisingly, a strong $\mathrm{MC}^{\perp}$ is obtained just after the threshold
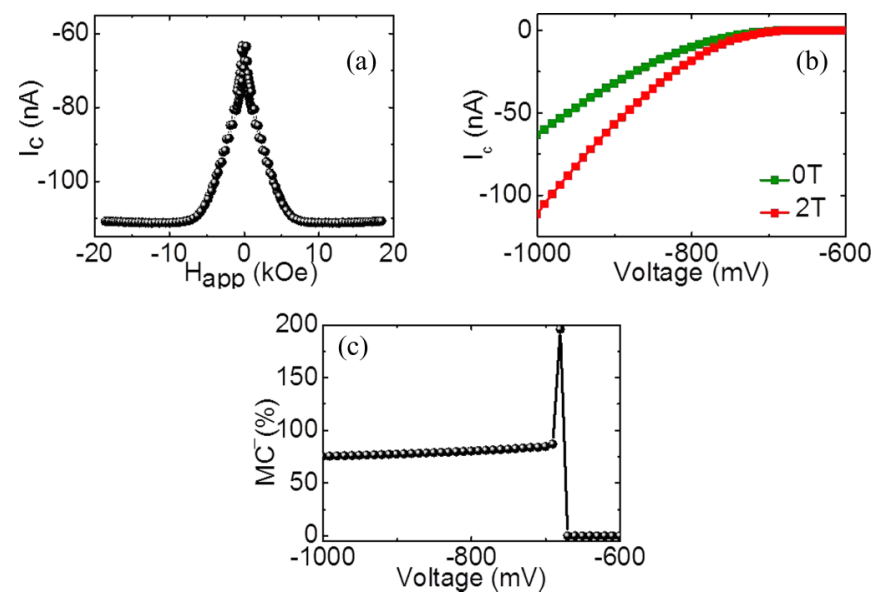

FIG. 2. Measurements done at $50 \mathrm{~K}$ on a $\mathrm{Si} / / \mathrm{Cu}(5) \mathrm{Ta}(1) \mathrm{Cu}(5)$ $\mathrm{Ni}(0.6)[\mathrm{Co}(0.2 / \mathrm{Ni}(0.6)] 4 \mathrm{Cu}(3.5) \mathrm{Co}(3) \mathrm{MgO}(2.8) \mathrm{Cu}(20) \mathrm{Pt}(5)$ MTT. (a) Variation of the collected current as a function of applied field, with the field applied in plane and applied voltage of $1 \mathrm{~V}$. (b) Current collected in $\mathrm{Si}$ as a function of the voltage applied for the parallel magnetic configuration (red curve) and for the crossed magnetic configuration (green curve) of the spin valve. (c) Crossed magnetocurrent as a function of the applied voltage calculated via the curves of the hot-electron current as a function of the applied voltage in the parallel and the crossed magnetic configurations of the spin valve. of the Schottky barrier with values close to those reported previously [7]. At $0.68 \mathrm{~V}$, the $\mathrm{MC}^{\perp}$ reaches $200 \%$ calculated using $I_{C}^{P}=-0.2035 \mathrm{nA}$ and $I_{C}^{\perp}=-0.0687 \mathrm{nA}$. Considering Eq. (3) that we will be introducing in the following, this value of $I_{C}^{\perp}$ is possible only if $I_{C}^{A P}$, the hot-electron current in this antiparallel configuration, is positive for this specific applied voltage. We carefully checked that the origin of this high $\mathrm{MC}^{\perp}$ is not linked to a spurious effect like a leakage current of the Schottky barrier (that would be detrimental to the magnetocurrent ratio value). The origin of this particular situation is still under investigation and is beyond the scope of this paper.

Since the MC is linked to the difference of mean-free path between electrons having their spin parallel or antiparallel to the magnetization of the ferromagnetic layer, the $\mathrm{MC}^{\perp}$ should increase with the layer thickness as observed previously in MTT with collinear magnetizations configurations. We have kept the Co thickness constant to $3 \mathrm{~nm}$ and varied $N$, the number of repetitions of the $[\mathrm{Co}(0.2) / \mathrm{Ni}(0.6)]_{N}$ multilayer. Inside the multilayer, the thicknesses of the Co and $\mathrm{Ni}$ layers have been kept constant. Since the stack $[\mathrm{Co}(0.2) / \mathrm{Ni}(0.6)]_{N}$ has perpendicular magnetization for $N$ between 1 and $5[8]$, the $\mathrm{MC}^{\perp}$ has been measured for five $\mathrm{Si} / / \mathrm{Cu}(5) \mathrm{Ta}(1) \mathrm{Cu}(5) \mathrm{Ni}(0.6)\left[\mathrm{Co}(0.2 / \mathrm{Ni}(0.6)]_{1-5} \mathrm{Cu}(3.5)\right.$ $\mathrm{Co}(3) \mathrm{MgO}(2.8) \mathrm{Cu}(20) \mathrm{Pt}(5)$ magnetic tunnel transistors. In Fig. 3(a), the variation of the $\mathrm{MC}^{\perp}$ as a function of the number of repetitions is shown for an applied voltage of $-1 \mathrm{~V}$. As expected, the $\mathrm{MC}^{\perp}$ increases with the number of repetitions. The highest value of $85 \%$ is measured for the MTT with five repetitions of the multilayer.

According to Ref. [9], the collected current can be expressed as (this will be confirmed in the last part of the paper)

$$
I_{C}=I_{C}^{\perp}\left(1+\mathrm{MC}^{\perp} \overrightarrow{u_{\mathrm{Co}}} \cdot \overrightarrow{u_{\mathrm{Co} / \mathrm{Ni}}}\right),
$$

in which $I_{C}^{\perp}$ is the current collected in a crossed configuration of the spin valve and $\mathrm{MC}^{\perp}$ is the crossed magnetocurrent defined in Eq. (1). $\overrightarrow{u_{\mathrm{Co}}}$ and $\overrightarrow{u_{\mathrm{Co} / \mathrm{Ni}}}$ are the unit vectors pointing in the directions of, respectively, the $\mathrm{Co}$ and $[\mathrm{Co} / \mathrm{Ni}]$ magnetization. The current in the crossed geometry is then given by

$$
I_{C}^{\perp}=\frac{1}{2}\left(I_{C}^{P}+I_{C}^{\mathrm{AP}}\right) .
$$

By rewriting Eq. (1), we get

$$
\begin{gathered}
\mathrm{MC}^{\perp}=100\left(\frac{I_{C}^{P}-I_{C}^{\mathrm{AP}}}{I_{C}^{\mathrm{AP}}}\right) \frac{1}{1+I_{C}^{P} / I_{C}^{\mathrm{AP}}}, \\
\mathrm{MC}^{\perp}=\mathrm{MC}^{\mathrm{Col}} \frac{1}{1+I_{C}^{P} / I_{C}^{\mathrm{AP}}},
\end{gathered}
$$

where $\mathrm{MC}^{\mathrm{Col}}$ is the magnetocurrent in the collinear magnetizations geometry when switching from parallel to antiparallel configuration. While $\mathrm{MC}^{\mathrm{Col}}$ can reach several thousand percent $[5,6]$, the $\mathrm{MC}^{\perp}$ saturates toward $100 \%$. With a measured maximum $\mathrm{MC}^{\perp}$ of $85 \%$ at applied voltage of $-1 \mathrm{~V}$ for the MTT with five repetitions of the multilayer, we are close to the ultimate value of $100 \%$ which can be obtained with our definition of the crossed magnetocurrent. This indicates a very large polarization efficiency through the high-quality spin-valve structure. 

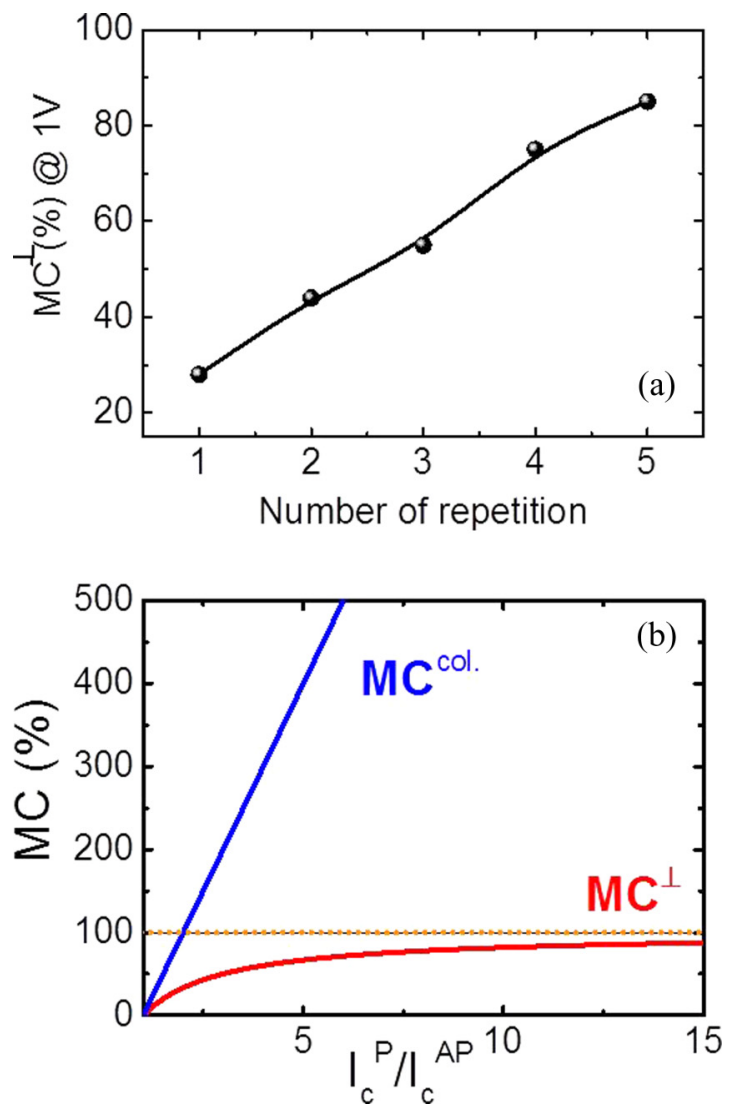

FIG. 3. (a) Crossed magnetocurrent $\mathrm{MC}^{\perp}$ as a function of the number of repetition of the $[\mathrm{Co} / \mathrm{Ni}]$ multilayer at an applied voltage of $-1 \mathrm{~V}$, for a $\mathrm{Si} / / \mathrm{Cu}(5) \mathrm{Ta}(1) \mathrm{Cu}(5) \mathrm{Ni}(0.6)$ $\left[\mathrm{Co}(0.2 / \mathrm{Ni}(0.6)]_{\mathrm{N}} \mathrm{Cu}(3.5) \mathrm{Co}(3) \mathrm{MgO}(2.8) \mathrm{Cu}(20) \mathrm{Pt}(5) \mathrm{MTT}\right.$. The temperature is $50 \mathrm{~K}$. (b) Calculated variation of the collinear magnetocurrent $\mathrm{MC}^{\mathrm{col}}$ and of the crossed magnetocurrent $\mathrm{MC}^{\perp}$ as a function of $I_{\mathrm{C}}^{\mathrm{P}} / I_{\mathrm{C}}^{\mathrm{AP}}$.

These conclusions are based on Eq. (2). In the last part of the paper, we will use the fact that our MTT has a crossed magnetization geometry to check the validity of Eq. (2). Indeed, our samples offer a unique opportunity to deepen our understanding on the angular dependence of the magnetocurrent since the relative angle between the magnetizations of the MTT layers can be controlled between $0^{\circ}$ and $90^{\circ}$. In the following, a $\mathrm{Si}[100](\mathrm{HF}) / / \mathrm{Cu}(5) \mathrm{Ta}(1) \mathrm{Cu}(5) \mathrm{Ni}(0.6)$ $\left[\mathrm{Co}(0.2 / \mathrm{Ni}(0.6)]_{4} \mathrm{Cu}(3.5) \mathrm{Co}(3) \mathrm{MgO}(2.8) \mathrm{Cu}(20) \mathrm{Pt}(5)\right.$ device has been used and an injection energy of $1 \mathrm{eV}$ has been used. The angles notation is given in Fig. 4.

In a first set of experiments, the collected current $I_{\mathrm{c}}$ has been measured either with the magnetic field applied out

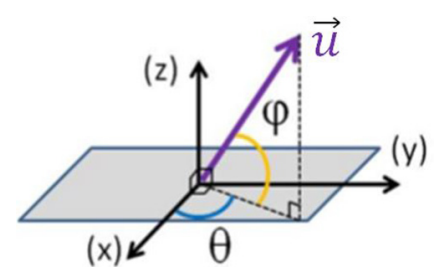

FIG. 4. Definition of the angles using for the experiments performed in the last part of the paper. $(X, Y)$ is the plane of the sample.

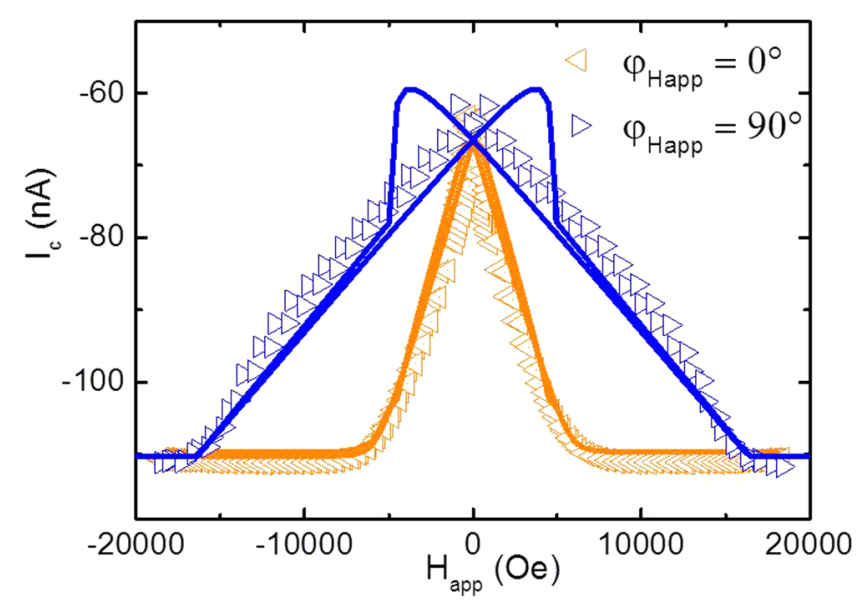

FIG. 5. $I_{\mathrm{c}}$ vs $H_{\text {app }}$ measured at $60 \mathrm{~K}$ and at $1 \mathrm{~V}$ of injection bias, with an in-plane field (orange curve) and an out-of-plane field (blue curve). Continuous lines correspond to fits performed using the model under discussion.

of the sample plane (OOP geometry) and so $\varphi_{H \text { app }}=90^{\circ}$ or with the magnetic field applied in plane (IP geometry), and so $\varphi_{H \text { app }}=0^{\circ}$. Figure 5 reports the obtained results in these two configurations. In both cases, the magnetizations of each layer are parallel and aligned along the magnetic field direction for a high field $(2 \mathrm{~T})$. The same value $I_{C}^{P}=-110 \mathrm{nA}$ of the collected current is obtained as expected. When the field is decreased to $0 \mathrm{~T}$, the magnetizations of both layers are orthogonal and the same value of the collected current is obtained again in both cases: $I_{C}^{\perp}=-65 \mathrm{nA}$. Thus, the $\mathrm{MC}^{\perp}$ is determined to be equal to $70 \%$.

We can reasonably state that the shapes of the curves are directly linked to the demagnetization field of the Co layer in the OOP geometry, and to the perpendicular anisotropy of the $\mathrm{Ni}(0.6)[\mathrm{Co}(0.2) / \mathrm{Ni}(0.6)] 4$ in the IP geometry.

To determine precisely these two values, it is required to

(1) Model the magnetic response of the system with respect to the magnetic field and thus to obtain values of $\overrightarrow{u_{\mathrm{Co}}} \cdot \overrightarrow{u_{\mathrm{Co} / \mathrm{Ni}}}$ vs $H_{\text {app. }}$.

(2) Use Eq. (2) to compute the collected current knowing the value of $\overrightarrow{u_{\mathrm{Co}}} \cdot \overrightarrow{u_{\mathrm{Co} / \mathrm{Ni}}}$.

The value of $\overrightarrow{u_{\mathrm{Co}}} \cdot \overrightarrow{u_{\mathrm{Co} / \mathrm{Ni}}}$ vs $H_{\text {app }}$ in both geometry IP and OOP has been determined thanks to numerical computation supposing that

(i) The magnetizations of both magnetic layers remain uniform regardless of the values and the directions of the applied field,

(i) The magnetizations of each layer are independent from each other (uncoupled magnetic system),

(i) Only a first-order uniaxial anisotropy is acting in each layer.

In this framework, we divide our problem into two uncoupled systems having each a volume energy density, normalized to saturation magnetization, of the form

$$
E_{\mathrm{Z}}+E_{\mathrm{A}}+E_{\mathrm{D}}
$$

where $E_{\mathrm{Z}}$ is the Zeeman energy equal to $-\overrightarrow{H_{\mathrm{app}}} \cdot \vec{u}, E_{\mathrm{A}}$ corresponds to the first-order uniaxial anisotropy expressed 
as $\frac{1}{2} H_{\text {an }} \sin ^{2}\left(\cos ^{-1}\left(\frac{\overrightarrow{H_{\text {an }}}}{H_{\mathrm{an}}} \cdot \vec{u}\right)\right), H_{\mathrm{an}}$ being the anisotropy field. The last term taking into account the demagnetization field is expressed as $H_{D} \sin ^{2}\left(\varphi_{u}\right)$, where $H_{\mathrm{D}}$ is the demagnetization field, and $\varphi_{\mathrm{u}}$ is the angle between the magnetization direction $\vec{u}$ and the sample plane, as defined in Fig. 4.

Each layer magnetization has only two degrees of freedom, denoted in the following: $\theta_{[\mathrm{Co} / \mathrm{Ni}]}$ and $\varphi_{[\mathrm{Co} / \mathrm{Ni}]}$ in the case of the $[\mathrm{Co} / \mathrm{Ni}]$ multilayer having an out-of-plane anisotropy axis and $\theta_{\mathrm{Co}}$ and $\varphi_{\mathrm{Co}}$ in the case of the Co layer with an in-plane anisotropy axis. We have computed, in a recursive manner, the stable state of magnetizations for each value of the field and in both geometries (IP and OOP).

Our best fits of the $I_{\mathrm{c}}$ vs $H_{\text {app }}$ curves are presented in Fig. 5 by the blue and orange continuous lines. This fitting procedure allows us to obtain a value of $0.55 \mathrm{~T}$ for the perpendicular anisotropy field of the $[\mathrm{Co} / \mathrm{Ni}]$ multilayer, and a value equal to $1.6 \mathrm{~T}$ for the demagnetizing field of the 3-nm-thick Co layer sandwiched between the $\mathrm{Cu}$ layer and the $\mathrm{MgO}$ tunnel barrier. It is about $10 \%$ lower than $1.8 \mathrm{~T}$, which is the expected value neglecting perpendicular anisotropy originating from the $\mathrm{MgO} / \mathrm{Co}$ interface. The obtained fits are well reproducing the measured curves except for the hysteretic parts present at low fields. This is certainly a limitation of the used model that supposes that the magnetization of each magnetic layer remains uniform during its reversal. This uniformity condition is probably not respected experimentally since the reversal of the $\mathrm{Ni}(0.6)[\mathrm{Co}(0.2) / \mathrm{Ni}(0.6)]_{4}$ magnetization in the OOP geometry is likely to take place by nucleation propagation. From this first step, the values of $\mathrm{I}^{\perp}, \mathrm{MC}^{\perp}, H_{\mathrm{D}}$, and $H_{\text {an }}$ have been obtained.

Then the collected current $I_{\mathrm{c}}$ has been measured as a function of $\varphi_{H \text { app }}$, the angle between the sample plane and the applied field. Figure 6 present measurements done for different magnitudes of the magnetic field (200, 1000, 3000, 5000 Oe). During these experiments, $\varphi_{H \text { app }}$ is increased from $-50^{\circ}$ to $+310^{\circ}$ and then decreased from $+310^{\circ}$ to $-50^{\circ}$. The collected current is measured at $60 \mathrm{~K}$ for an injection bias of $-1 \mathrm{~V}$. At 200 Oe (blue curve), a reversible signal having mostly a cosine signature has been recorded. The magnetic-field increase is

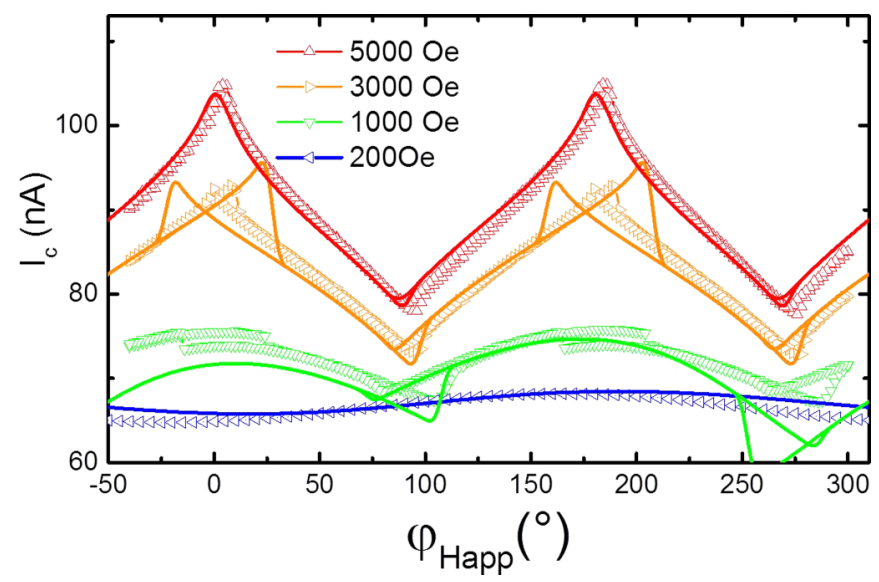

FIG. 6. Collected current $I_{\mathrm{c}}$ as a function of the field angle $\varphi_{H \text { app }}$, measured at $60 \mathrm{~K}$ and for an applied voltage $V_{\mathrm{e}}=-1 \mathrm{~V}$. The magnitude of the applied field is 200, 1000, 3000, or 5000 Oe. Continuous lines correspond to fits performed using the model under discussion.
TABLE I. Set of used parameters to reproduce our measurements. Parameters extracted from the $I_{\mathrm{c}}$ vs $H_{\text {app }}$ fitting procedure are presented in green; the parameters used for the experimental angular dependence fitting are presented in black.

\begin{tabular}{|l|l|l|}
\hline & $\overrightarrow{u_{C o / N \imath}}$ & $\overrightarrow{u_{C o}}$ \\
\hline $\mathrm{H}_{\mathrm{an}}$ & $0,55 \mathrm{~T}$ & $0,04 \mathrm{~T}$ \\
\hline$\theta_{\text {Han }}$ & $0^{\circ}$ & $5^{\circ}$ \\
\hline$\varphi_{\text {Han }}$ & $92^{\circ}$ & $0^{\circ}$ \\
\hline $\mathrm{H}_{\mathrm{D}}$ & 0 & $1,6 \mathrm{~T}$ \\
\hline
\end{tabular}

\begin{tabular}{|l|l|}
\hline$\theta_{\text {Happ }}$ & $10^{\circ}$ \\
\hline $\mathrm{I}^{\perp}$ & $-65 \mathrm{nA}$ \\
\hline $\mathrm{MC}^{\perp}$ & 0,7 \\
\hline
\end{tabular}

accompanied by both the appearance of irreversible (hysteric) parts and the loss of the cosine shape.

By using the values of $\mathrm{I}^{\perp}, \mathrm{MC}^{\perp}, H_{\mathrm{D}}$, and $H_{\text {an }}$ previously obtained, we attended to compute the angular measurements. The values of $\theta_{[\mathrm{Co} / \mathrm{Ni}]}, \varphi_{[\mathrm{Co} / \mathrm{Ni}]}, \theta_{\mathrm{Co}}$, and $\varphi_{\mathrm{Co}}$ are determined by minimizing the energies of two independent subsystems for each value of $\varphi_{H \text { app }}$. This enables then the calculation of $I_{\mathrm{c}}\left(\varphi_{H \text { app }}\right)$ thanks to Eq. (2). Our fits of the experimental data are presented by the continuous lines in Fig. 6. Table I presents the parameters used to reproduce our measurements.

This set of parameters does not contain any surprising value. A slight misalignment between the sample anisotropies axes and the magnetic field direction has to be introduced in order to obtain a reasonable agreement between the computed signals and the measurements (see nonzero values of $\theta_{H \text { an }}, \varphi_{H \text { an }}$, and $\left.\theta_{H \text { app }}\right)$. Again, the magnetic part of the model failed to reproduce quantitatively the irreversible parts. As previously, we attribute this deviation to the nonuniform magnetic reversals occurring experimentally. However, it is remarkable that the model well captures the shape variation of our angular measurement when increasing the applied magnetic field. Furthermore, the variation of the base current with the field magnitude is also very well reproduced.

\section{CONCLUSION}

In summary, by taking advantage of the crossed geometry, we demonstrate that we are able to reproduce both current vs field direction and current vs field intensity measurements in a framework taking into account a reduced number of parameters and a simple cosine dependence of the hot-electron current on the angle between magnetizations. Furthermore, as for MTTs with collinear magnetic configurations, we report the magnetocurrent as a function of the magnetic layer thickness that increases with layer thickness up to $85 \%$, slightly lower than $100 \%$, the maximum theoretical magnetocurrent that can be reached in the crossed magnetizations configuration.

\section{ACKNOWLEDGMENTS}

This work has been done partially in the framework of the SpinPress ANR Project. Experiments were carried out on IJL Project TUBE-Davm equipments funded by FEDER (EU), French PIA project Lorraine Université d'Excellence (Grant No. ANR-15-IDEX-04-LUE), Region Grand Est, Metropole Grand Nancy, and ICEEL. 
[1] D. J. Monsma, J. C. Lodder, Th. J. A. Popma, and B. Dieny, Phys. Rev Lett. 74, 5260 (1995).

[2] K. Mizushima, T. Kinno, K. Tanaka, and T. Yamauchi, IEEE Trans. Magn. 33, 3500 (1997).

[3] R. Sato and K. Mizushima, Appl. Phys. Lett. 79, 1157 (2001).

[4] S. Van Dijken, X. Jiang, and S. S. P. Parkin, Appl. Phys. Lett. 83, 951 (2003).
[5] Y. Lu, D. Lacour, G. Lengaigne, S. Le Gall, S. Suire, F. Montaigne, and M. Hehn, Appl. Phys. Lett. 103, 022407 (2013).

[6] R. Jansen, J. Phys. D 36, R289 (2003).

[7] C. Vautrin, Y. Lu, S. Robert, G. Sala, O. Lenoble, S. Petit-Watelot, X. Devaux, F. Montaigne, D. Lacour, and M. Hehn, J. Phys. D 49, 355003 (2016).

[8] C. Vautrin, Ph.D. thesis, Lorraine University (2017).

[9] I. Appelbaum, Philos. Trans. R. Soc. A 369, 3554 (2011). 